\title{
Dual energy computed tomography in differentiation of iodine contrast agent staining from secondary brain haemorrhage in patients with ischaemic stroke treated with thrombectomy
}

\author{
Robert Chrzan ${ }^{1}$, Bartłomiej Łasocha ${ }^{2}$, Paweł Brzegowy ${ }^{1}$, Tadeusz Popiela ${ }^{1}$ \\ ${ }^{1}$ Department of Radiology, Jagiellonian University Medical College, Krakow, Poland \\ ${ }^{2}$ Diagnostic Imaging Unit, University Hospital, Krakow, Poland
}

\begin{abstract}
Introduction. The aim of this study was to assess the value of dual energy computed tomography (DECT) in the differentiation of iodine contrast agent staining from secondary brain haemorrhage in patients with acute ischaemic stroke treated with mechanical thrombectomy.

Material and methods. The group analysed consisted of 66 hyperdense areas in 64 patients with acute ischaemic stroke treated with mechanical thrombectomy and controlled in DECT performed within the first 24 hours after thrombectomy. In every area both qualitative and quantitative analysis of iodine and water material density (MD) maps was performed for the differentiation of iodine and blood, as well as CT density analysis.

Results. $66.7 \%$ of hyperdense areas were classified as iodine, $18.2 \%$ as iodine + blood, and $15.1 \%$ as blood. The density of iodine was significantly higher in the iodine (median $9.64100 \mathrm{ug} / \mathrm{cm} 3$ ) group compared to the blood (median $3.97100 \mathrm{ug} / \mathrm{cm} 3$ ) and iodine + blood (median $7.57100 \mathrm{ug} / \mathrm{cm} 3$ ) groups. The density of water was significantly higher in the blood (median 1,051.50 $\mathrm{mg} / \mathrm{cm} 3$ ) and iodine + blood (median 1,038.00 mg/cm3) groups compared to the iodine (median 1,021.00 mg/cm3) group.

Conclusions. DECT with iodine-water material decomposition maps is a valuable tool in the differentiation of prolonged staining of iodine contrast agent from secondary brain haemorrhage in patients with acute ischaemic stroke treated with mechanical thrombectomy. The value of $6100 \mathrm{ug} / \mathrm{cm} 3(0.6 \mathrm{mg} / \mathrm{cm} 3)$ seems a good threshold in quantitative differentiation of iodine from blood on iodine (water) MD maps. The value of $1,030 \mathrm{mg} / \mathrm{cm} 3$ seems a good threshold in quantitative differentiation of iodine from blood on water (iodine) MD maps.
\end{abstract}

Key words: dual energy computed tomography, brain haemorrhage, ischaemic stroke, thrombectomy

(Neurol Neurochir Pol 2022; 56 (1): 68-74)

\section{Introduction}

Computed tomography (CT) brain examination is still the most commonly used imaging modality in patients with symptoms of acute brain stroke [1-3]. The method is fast, widely available, and inexpensive. It is also performed as a basic procedure to control patients after stroke treatment including intravenous thrombolysis and mechanical thrombectomy. One of the most important goals of a follow-up evaluation is to exclude secondary intracranial haemorrhage, which is still an important complication $[4,5]$.

Dual energy CT (DECT), also known as spectral CT, is a CT technique using two separate $\mathrm{X}$-ray photon energy spectra, which allows the distinction of materials with different

Address for correspondence: Robert Chrzan, Department of Radiology, Jagiellonian University Medical College, Kopernika 19, 31-501 Krakow, Poland; e-mail: robert.chrzan@uj.edu.pl

Received: 7.08.2021 Accepted: 19.10.2021 Early publication date: 5.01.2022

This article is available in open access under Creative Common Attribution-Non-Commercial-No Derivatives 4.0 International (CC BY-NC-ND 4.0) license, allowing to download articles and share them with others as long as they credit the authors and the publisher, but without permission to change them in any way or use them commercially. 
attenuation properties at different energies. This enables reconstruction of additional types of images: virtual monoenergetic images (simulating attenuation at a single photon energy rather than a spectrum), material decomposition images (mapping of substances of known attenuation characteristics), virtual non-contrast images (iodine virtually removed), iodine concentration (iodine maps), and electron density maps or effective atomic number (Zeff) maps [6, 7].

There are many DECT technologies available from different vendors, including: dual-source, single-source consecutive, single-source twin-beam, single-source sequential, single-source rapid kilovoltage switching, and dual-layer (so-called 'sandwich').

Gemstone Spectral Imaging (GSI) is a General Electric (GE) proprietary dual energy scan technique using rapid switching between low $\mathrm{kVp}$ and high $\mathrm{kVp}$ energies. This enables raw data-based reconstruction of dual energy data with the associated benefits of quantitative material decomposition. Based on known attenuation curves, the software mathematically transforms low and high $\mathrm{kVp}$ attenuation measurements into effective material density (MD) basis-pair images. It is also known as material decomposition, and it provides additional capabilities for CT analysis. Selection of MD pairs is based on the clinical question being investigated and materials of interest, such as iodine-water, iodine-calcium, or water-calcium. Such data processing is not possible using conventional CT scanners.

For example, both iodine contrast medium and fresh blood are hyperdense on conventional CT images. However, DECT and iodine-water material decomposition will generate two sets of images: iodine (water) with bright areas representing iodine, and water (iodine) with bright areas representing blood.

In patients treated with thrombectomy, a significant volume of iodine contrast medium is used during digital subtraction angiography (DSA). Due to damage to the blood-brain barrier in the stroke area, and potential endothelial damage during thrombectomy, this may result in a prolonged staining of the contrast medium, presenting in conventional control CT as a hyperdense region, just the same as is seen in secondary haemorrhage [8-10].

However, when performing DECT and iodine-water material decomposition, it should be possible to distinguish iodine staining from brain haemorrhage on MD maps. This is critical for the applicability of antithrombotic treatment in ischaemic stroke [10-12].

The aim of this pilot study was to assess the value of dual energy computed tomography in the differentiation of iodine contrast agent staining from secondary brain haemorrhage in patients with acute ischaemic stroke treated with mechanical thrombectomy.

\section{Material and methods}

The group consisted of retrospectively analysed 64 consecutive patients with acute cerebral ischaemic stroke ( 37 females and 27 males, aged 28-90 years, mean 69), treated with mechanical thrombectomy in the University Hospital in Krakow in 2020-2021, presenting hyperdense areas suspected of secondary haemorrhage in follow-up CT performed within the first 24 hours after thrombectomy. A single hyperdense area was visualised in 62 patients, and two independent areas in two patients. Therefore, 66 areas were finally analysed in the 64 patients.

In Digital Subtraction Angiography (DSA) during thrombectomy, a thrombus was located in the right middle cerebral artery in 24 cases, left middle cerebral artery in 18, right internal carotid artery in six, left internal carotid artery in six, basilar artery in three, both in the right middle cerebral artery and the right anterior cerebral artery in two, left posterior cerebral artery in two, right common carotid artery in one, left common carotid artery in one, and right vertebral artery in one case.

In all the patients, CT was performed using a 64-raw GE Revolution HD or a 256-raw GE Revolution CT scanner and GSI dual energy protocol with rapid $\mathrm{kV}$ switching. The CT protocol parameters were as follows: detector coverage $20 \mathrm{~mm}$, slice thickness $2.5 \mathrm{~mm}$, pitch 0.5 , rotation time $0.7 \mathrm{~s}$, current $315-375 \mathrm{~mA}$, voltage $80 \mathrm{kVp} / 140 \mathrm{kVp}$. These parameters were selected from the range of GE presets to get the final GSI $\mathrm{X}$-ray dose similar to the dose in conventional head CT - the obtained CTDIvol value was 53-60 mGy.

In every case, in addition to the typical CT greyscale images, two sets of MD maps were generated using GE CT scanner software: iodine (water) and water (iodine). It should be emphasised that MD maps are not scaled in Hounsfield units (HU) like typical CT images, but in absolute values: 100ug/ $\mathrm{cm} 3$ of iodine on the iodine (water) map and $\mathrm{mg} / \mathrm{cm} 3$ of water on the water (iodine) map.

In every hyperdense area:

- qualitative analysis of MD maps was performed as for the differentiation of iodine and blood, using grouping terms: iodine (only iodine or much more iodine than blood), iodine + blood (mixed comparable volumes of iodine and blood), and blood (only blood or much more blood than iodine);

- quantitative analysis of MD maps was performed using region of interest (ROI) measurements in the locations corresponding to the hyperdense areas on CT greyscale images;

- CT density analysis in HU was performed using ROI measurements in the hyperdense areas on CT images.

The analysis was performed by a diagnostic neuroradiologist with 25 years of experience (RC) and an interventional radiologist with eight years of experience $(\mathrm{B} \ell)$. In qualitative analysis, both of the radiologists reviewed the images, and any disagreement was resolved by consensus. In quantitative analysis, the ROI measurements were performed by a diagnostic neuroradiologist, with two measurements of each value and the mean value as the result. 


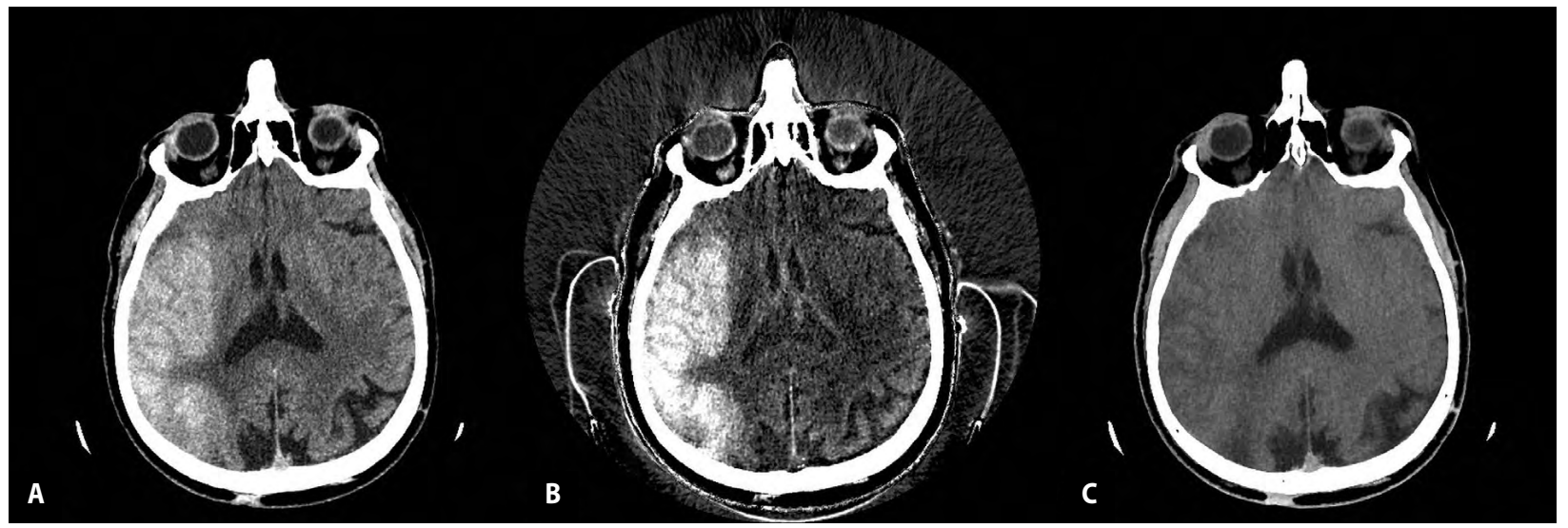

Figure 1. Hyperdense area on CT image, corresponding to iodine on MD maps; A. CT image; B. lodine (water) MD map; C. Water (iodine) MD map

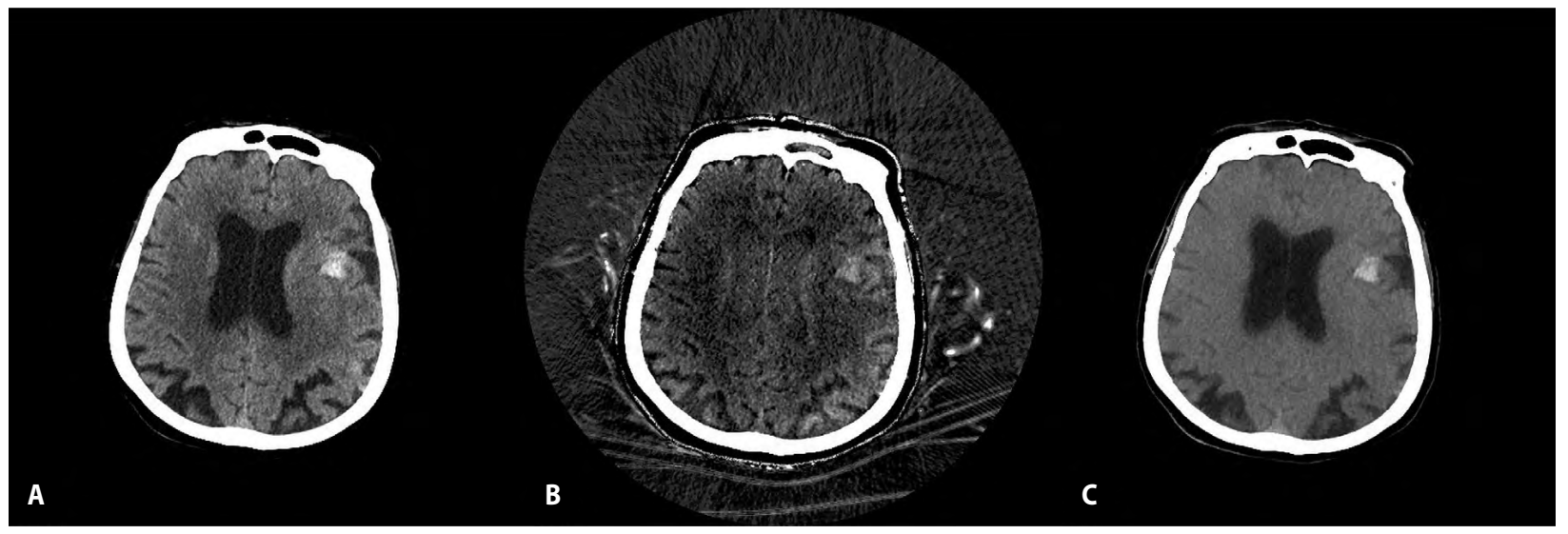

Figure 2. Hyperdense area on CT image, corresponding to iodine and blood on MD maps; A. CT image; B. lodine (water) MD map; C. Water (iodine) MD map

Statistica 13.3 software (TIBCO Software Inc., Palo Alto, CA, USA) was used for statistical computations.

Due to the lack of confirmation of the normal distribution in the Shapiro-Wilk's test, the comparison of the values of quantitative variables in three qualitative groups was performed using the Kruskal-Wallis test. Post-hoc analysis with Dunn's test was performed to identify significantly different groups.

Patient consent was waived due to the retrospective assessment of CT images, obtained as a part of routine thrombectomy follow-up protocol.

\section{Results}

In all the patients, the follow-up CT was performed within the first 24 hours after thrombectomy. In 14 patients, the date of CT was the same as the date of thrombectomy, and in 50 patients the date of CT was the day after thrombectomy.

In qualitative analysis of MD maps, $44(66.7 \%)$ areas were classified as iodine (Fig. 1), 12 (18.2\%) as iodine + blood (Fig. 2), and 10 (15.1\%) as blood (Fig. 3).
In quantitative analysis of iodine (water) MD maps, the density of iodine was significantly higher in the iodine (median $9.64100 \mathrm{ug} / \mathrm{cm} 3$ ) group compared to the blood (median $3.97100 \mathrm{ug} / \mathrm{cm} 3)$ and iodine + blood (median $7.57100 \mathrm{ug} / \mathrm{cm} 3)$ groups (Fig. 4). The value of $6100 \mathrm{ug} / \mathrm{cm} 3(0.6 \mathrm{mg} / \mathrm{cm} 3)$ seems a good threshold in quantitative differentiation of iodine from blood on iodine (water) MD maps.

In quantitative analysis of water (iodine) MD maps, the density of water was significantly higher in the blood (median $1,051.50 \mathrm{mg} / \mathrm{cm} 3$ ) and iodine + blood (median 1,038.00 mg/ $\mathrm{cm} 3$ ) groups compared to the iodine (median $1,021.00 \mathrm{mg} /$ $\mathrm{cm} 3$ ) group (Fig. 5). The value of $1,030 \mathrm{mg} / \mathrm{cm} 3$ seems a good threshold in quantitative differentiation of iodine from blood on water (iodine) MD maps.

In the analysis of CT images, no significant differences of $\mathrm{CT}$ density in $\mathrm{HU}$ were found between the groups i.e. iodine, iodine + blood, and blood (Fig. 6). This confirms that the differentiation of iodine from blood was not possible using only CT images without MD maps.

The results are summarised in Table 1. 


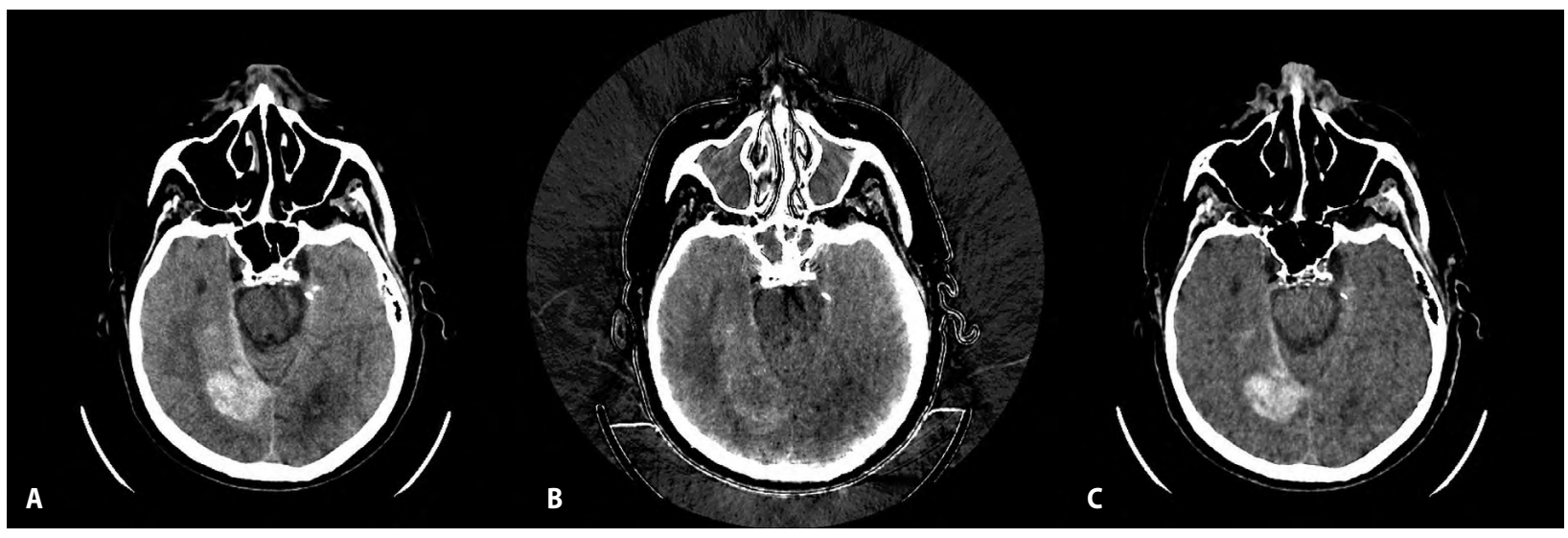

Figure 3. Hyperdense area on CT image, corresponding to blood on MD maps; A. CT image; B. lodine (water) MD map; C. Water (iodine) MD map



Figure 4. Comparison of iodine density on iodine (water) MD maps in three subgroups: iodine, iodine + blood, and blood; MD - material density

\section{Discussion}

The most commonly used method of differentiation contrast staining from blood after thrombectomy in CT is re-examination within three days [8-10]. Spontaneous resolution of iodine staining but not haemorrhage is then observed in the next CT examination. However, this may delay secondary thromboprophylaxis.

Also, MRI can be used to distinguish iodine from blood, typically using gradient recalled-echo sequences [13, 14]. However, access to MRI may be limited by logistical considerations or contraindications.
DECT seems to provide a much better alternative, avoiding the problems related to CT follow-up and MRI assessment.

In the study by Liu et al. [15], DECT was performed in 106 patients immediately and 24 hours after thrombectomy, and conventional non-contrast CT was performed three days after thrombectomy. In DECT immediately after thrombectomy, $64.2 \%$ of cases were classified as pure iodine contrast, $10.4 \%$ as haemorrhage mixed with iodine contrast, and $0 \%$ as haemorrhage only; the remaining $25.4 \%$ showed no hyperdensities. However, in DECT $24 \mathrm{~h}$ after thrombectomy, these values changed to $11.3 \%$ of pure iodine contrast, $16.0 \%$ of haemorrhage mixed with iodine contrast, $14.2 \%$ 


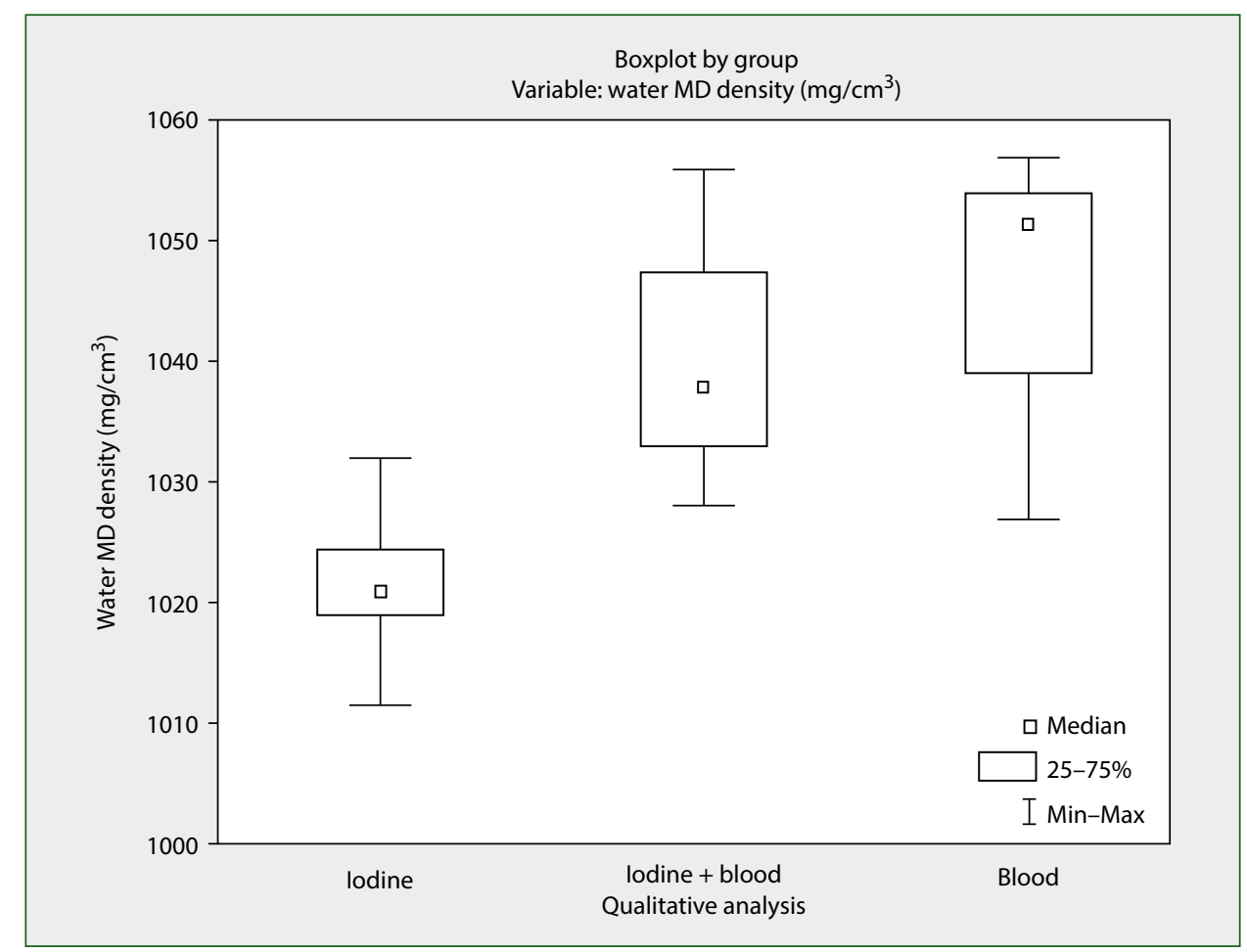

Figure 5. Comparison of water density on water (iodine) MD maps in three subgroups: iodine, iodine + blood, and blood; MD - material density

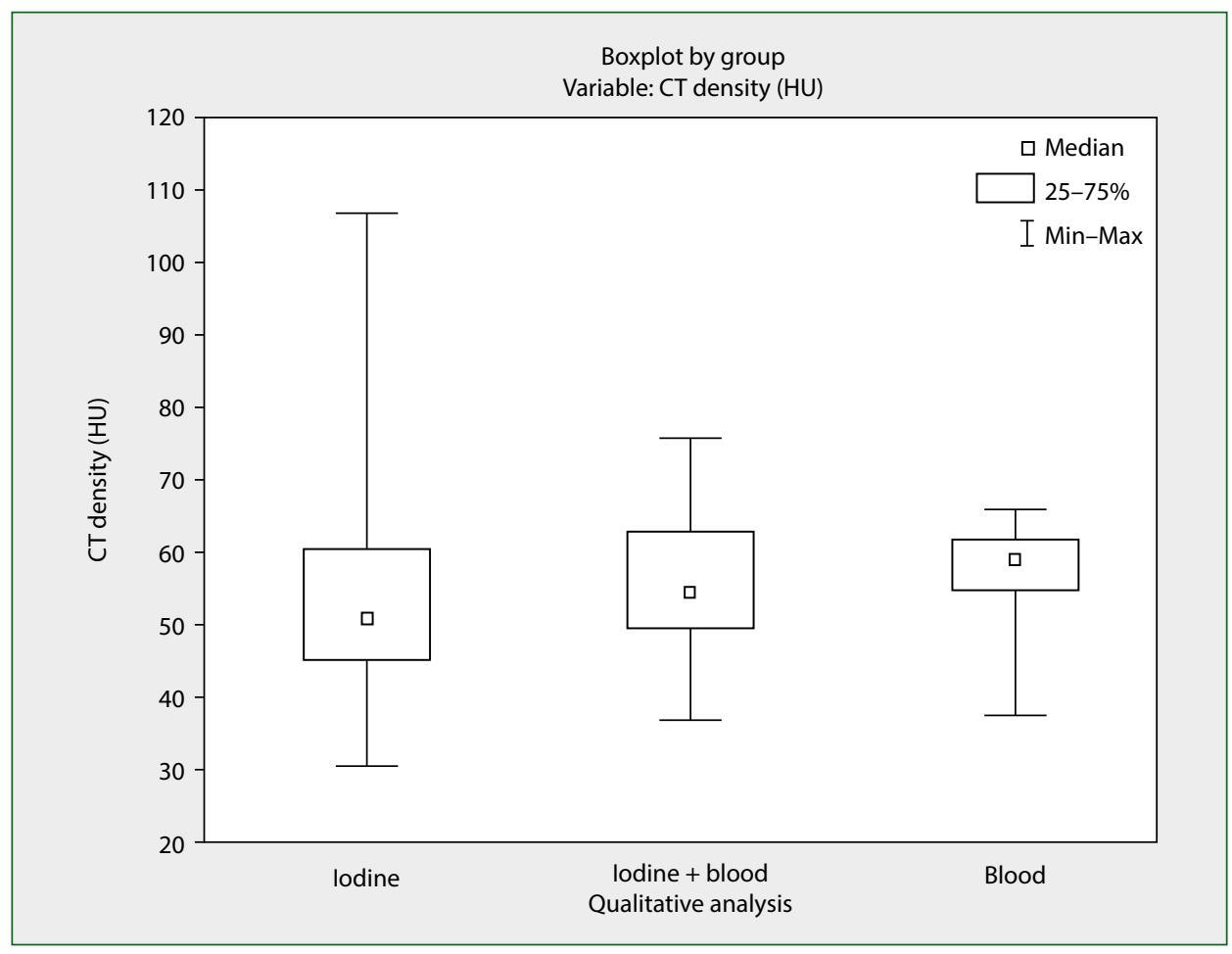

Figure 6. Comparison of CT density on CT images in three subgroups: iodine, iodine + blood, and blood; CT - computed tomography

of hemorrhage only, and $58.5 \%$ of no hyperdensities. This means that iodine staining was much more frequent just after thrombectomy and gradually subsided over the following hours. Contrastingly, the risk of haemorrhage increased within the first 24 hours. Liu et al. also found that the haemorrhagic transformation classified on DECT $24 \mathrm{~h}$ after thrombectomy was quite consistent with the conventional non-contrast CT after three days. 
Table 1. Results of statistical analysis of parameters: lodine density, water density on MD maps, CT density on CT images, in three groups: iodine, iodine + blood, and blood

\begin{tabular}{|c|c|c|c|c|c|}
\hline \multirow[t]{2}{*}{ Parameter } & & \multicolumn{3}{|c|}{ Group } & \multirow[t]{2}{*}{ P-value } \\
\hline & & $\begin{array}{l}\text { lodine } \\
(N=44)\end{array}$ & $\begin{array}{l}\text { lodine + blood } \\
\qquad(\mathbf{N}=12)\end{array}$ & $\begin{array}{c}\text { Blood } \\
(N=10)\end{array}$ & \\
\hline \multirow{3}{*}{$\begin{array}{l}\text { lodine density on } \\
\text { iodine (water) MD maps } \\
\text { (100ug/cm3) }\end{array}$} & Mean \pm SD & $10.97 \pm 4.81$ & $7.05 \pm 2.01$ & $4.03 \pm 1.31$ & \multirow{3}{*}{$\begin{array}{l}\text { lodine }>\text { blood } \\
p=0.000001 \\
\text { lodine }>\text { iodine }+ \text { blood } \\
p<0.05\end{array}$} \\
\hline & Median & 9.64 & 7.57 & 3.97 & \\
\hline & Quartiles & $7.33-13.04$ & $5.66-8.15$ & $3.01-4.76$ & \\
\hline \multirow{3}{*}{$\begin{array}{l}\text { Water density on } \\
\text { water (iodine) MD maps } \\
\text { (mg/cm3) }\end{array}$} & Mean \pm SD & $1,021.59 \pm 4.65$ & $1,039.83 \pm 9.13$ & $1,046.50 \pm 10.11$ & \multirow{3}{*}{$\begin{array}{l}\text { Blood }>\text { iodine } \\
p=0.000001 \\
\text { lodine }+ \text { blood }>\text { iodine } \\
p=0.000005\end{array}$} \\
\hline & Median & $1,021.00$ & $1,038.00$ & $1,051.50$ & \\
\hline & Quartiles & $1,019.00-1,024.50$ & $1,033.00-1,047.50$ & $1,039.00-1054.00$ & \\
\hline \multirow{3}{*}{$\begin{array}{l}\text { CT density on } \\
\text { CT images } \\
(\mathrm{HU})\end{array}$} & Mean \pm SD & $53.98 \pm 14.81$ & $56.25 \pm 11.80$ & $56.70 \pm 8.45$ & \multirow{3}{*}{$\begin{array}{l}\text { No significant } \\
\text { differences between } \\
\text { lodine } \\
\text { lodine + blood } \\
\text { Blood }\end{array}$} \\
\hline & Median & 51.00 & 54.50 & 59.50 & \\
\hline & Quartiles & $45.50-60.50$ & $50.00-63.00$ & $55.00-62.00$ & \\
\hline
\end{tabular}

The recalculated Liu results of DECT after $24 \mathrm{~h}$, including only 44 patients with hyperdensities as in our study, are: $27.3 \%$ of pure iodine contrast, $38.6 \%$ of haemorrhage mixed with iodine contrast, and $34.1 \%$ of haemorrhage only.

In our study, compared to Liu we found a much higher percentage of iodine contrast (66.7\%) relative to iodine mixed with blood (18.2\%) and blood only (15.1\%). The explanation for this may be performing DECT "within $24 \mathrm{~h}$ " in our study but "after 24 h" in Liu et al.s study.

Almqvist et al. [16] investigated whether DECT using a non-contrast CT, a water-weighted image after iodine removal, and an iodine-weighted image changes the diagnosis and classification of intracranial haemorrhage (ICH) after endovascular thrombectomy (EVT) in acute ischaemic stroke compared to a CT image alone, in a group of 372 patients. The use of DECT changed the ICH diagnosis to iodine contrast staining in $34 \%$ of cases, modified the ICH grade in $10 \%$, and diagnosed initially undetected ICH in $2 \%$. Almqvist et al. concluded this could affect decision-making regarding monitoring, secondary prevention, and prognostication.

In our study, we performed no independent analysis of 'conventional' CT and DECT as for ICH diagnosis, because we found no significant differences of CT density in HU in hyperdense areas on CT images. Perhaps such analysis is possible considering the shape and texture of hyperdensities - we plan to include it in future studies on a larger group.

Tijssen et al. [17] used DECT and iodine maps in a small group of 30 patients controlled by CT after mechanical revascularisation in acute ischaemic stroke. In a subgroup of 22 patients with hyperdense areas on conventional CT images, iodine maps suggested contrast extravasation in 18 patients; in $16 / 18$ patients this was confirmed at follow-up.

In a recent meta-analysis, Chen et al. [18] concluded that DECT shows excellent accuracy and specificity in differentiating intracerebral haemorrhage from iodine contrast extravasation after thrombectomy for acute ischaemic stroke. Nevertheless, there has been substantial heterogeneity among the studies, meaning that further large-scale, prospective studies are needed.

In our study, we presented the suggested thresholds in quantitative differentiation of iodine from blood on MD maps. Optimally, such thresholds should be based on receiver operating characteristic (ROC) analysis with area under curve calculations. However, in our analysis of MD maps, three groups were included (iodine, iodine + blood, and blood), whereas for ROC analysis we would need a test dividing all the cases into only two groups (iodine and blood). Therefore, our thresholds should be treated only as suggestions. We found no data in the available literature allowing for comparison of our thresholds with the values obtained by other authors. Bonatti [19] found a cutoff value of $1.35 \mathrm{mg} / \mathrm{mL}$ iodine concentration on iodine MD maps identifying patients developing ICH with $100 \%$ sensitivity and $47.5 \%$ specificity, but in his study DECT was performed immediately after mechanical thrombectomy.

Of course, differentiation of iodine staining from haemorrhage in DECT is not limited to patients treated by thrombectomy. For example, patients after stroke thrombolysis may be assessed in the same way. In another study by Almqvist et al. [20], 9.7\% of cases diagnosed as ICH in CT after thrombolysis changed to iodine staining in DECT.

The key limitation of our study is the lack of a reference standard, including the next follow-up non-contrast CT or susceptibility-weighted MRI, performed about three days post thrombectomy. This could significantly bias the results. Moreover, as a consequence, we could not assess the diagnostic accuracy, sensitivity, specificity, PPV or NPV of the test. Other limitations include its retrospective research nature (although we prospectively collected data using a stroke registry) and the limited number of cases. Therefore, we plan to conduct a prospective study based on a larger group of patients and including follow-up CT three days after thrombectomy. 
In summary, conventional non-contrast CT alone should be used with caution for the diagnosis of haemorrhage after mechanical thrombectomy, because iodine staining can mimic haemorrhage. DECT seems to offer a valuable alternative for early differentiation of haemorrhage and iodine contrast medium, facilitating clinical decision making regarding antithrombotic therapy.

\section{Conclusions}

Dual energy computed tomography with iodine-water material decomposition maps is a valuable tool in the differentiation of prolonged staining of iodine contrast agent from secondary brain haemorrhage in patients with acute ischaemic stroke treated with mechanical thrombectomy.

We suggest a value of $6100 \mathrm{ug} / \mathrm{cm} 3(0.6 \mathrm{mg} / \mathrm{cm} 3)$ as a good threshold in quantitative differentiation of iodine from blood on iodine (water) MD maps.

We suggest a value of $1,030 \mathrm{mg} / \mathrm{cm} 3$ as a good threshold in quantitative differentiation of iodine from blood on water (iodine) MD maps.

Conflict of interest: None.

Funding: None.

\section{References}

1. Expert Group of Vascular Diseases Section of Polish Neurological Society. Management of acute stroke - guidelines from the Expert Group of the Section of Cerebrovascular Diseases of the Polish Neurological Society. Neurol Neurochir Pol. 2012; 46(Suppl 1): 1-114.

2. Członkowska A, Kobayashi A. Expert Group of Vascular Diseases Section of Polish Neurological Society. Management of acute stroke guidelines from the Expert Group of the Section of Cerebrovascular Diseases of the Polish Neurological Society. Update 2013: thrombolysis. Neurol Neurochir Pol. 2013; 47(4): 303-309.

3. Jauch EC, Saver JL, Adams HP, et al. American Heart Association Stroke Council, Council on Cardiovascular Nursing, Council on Peripheral Vascular Disease, Council on Clinical Cardiology. Guidelines for the early management of patients with acute ischemic stroke: a guideline for healthcare professionals from the American Heart Association/ American Stroke Association. Stroke. 2013; 44(3): 870-947, doi: 10.1161/STR.0b013e318284056a, indexed in Pubmed: 23370205.

4. Khatri $P$, Wechsler LR, Broderick JP. Intracranial hemorrhage associated with revascularization therapies. Stroke. 2007; 38(2): 431-440, doi: 10.1161/01.STR.0000254524.23708.c9, indexed in Pubmed: 17234988.

5. Salsano G, Pracucci G, Mavilio N, et al. Complications of mechanical thrombectomy for acute ischemic stroke: Incidence, risk factors, and clinical relevance in the Italian Registry of Endovascular Treatment in acute stroke. Int J Stroke. 2021; 16(7): 818-827, doi: 10.1177/1747493020976681, indexed in Pubmed: 33283685.

6. Johnson T, Fink C, Schönberg S, Reiser M. Dual Energy CT in Clinical Practice. Springer-Varlag, Berlin 2011.

7. McCollough $\mathrm{CH}$, Leng S, Yu L, et al. Dual- and multi-energy CT: principles, technical approaches, and clinical applications. Radiology. 2015; 276(3): 637-653, doi: 10.1148/radiol.2015142631, indexed in Pubmed: 26302388.
8. Lummel N, Schulte-Altedorneburg G, Bernau C, et al. Hyperattenuated intracerebral lesions after mechanical recanalization in acute stroke. AJNR Am J Neuroradiol. 2014; 35(2): 345-351, doi: 10.3174/ajnr. A3656, indexed in Pubmed: 23907245.

9. Payabvash S, Qureshi MH, Khan SM, et al. Differentiating intraparenchymal hemorrhage from contrast extravasation on post-procedural noncontrast $\mathrm{CT}$ scan in acute ischemic stroke patients undergoing endovascular treatment. Neuroradiology. 2014; 56(9): 737-744, doi: 10.1007/s00234-014-1381-8, indexed in Pubmed: 24925217.

10. Yedavalli V, Sammet S. Contrast extravasation versus hemorrhage after thrombectomy in patients with acute stroke. J Neuroimaging. 2017; 27(6): 570-576, doi: 10.1111/jon.12446, indexed in Pubmed: 28514045.

11. Gupta R, Phan CM, Leidecker C, et al. Evaluation of dual-energy CT for differentiating intracerebral hemorrhage from iodinated contrast material staining. Radiology. 2010; 257(1): 205-211, doi: 10.1148/ radiol.10091806, indexed in Pubmed: 20679449.

12. Phan CM, Yoo AJ, Hirsch JA, et al. Differentiation of hemorrhage from iodinated contrast in different intracranial compartments using dual-energy head CT. AJNR Am J Neuroradiol. 2012; 33(6): 1088-1094, doi: 10.3174/ajnr.A2909, indexed in Pubmed: 22268092.

13. Nikoubashman O, Jablawi F, Dekeyzer S, et al. MRI appearance of intracerebral iodinated contrast agents: is it possible to distinguish extravasated contrast agent from hemorrhage? AJNR Am J Neuroradiol. 2016; 37(8): 1418-1421, doi: 10.3174/ajnr.A4755, indexed in Pubmed: 27032975.

14. You SH, Kim B, Kim BK, et al. MR imaging for differentiating contrast staining from hemorrhagic transformation after endovascular thrombectomy in acute ischemic stroke: phantom and patient study. AJNR Am J Neuroradiol. 2018; 39(12): 2313-2319, doi: 10.3174/ajnr. A5848, indexed in Pubmed: 30385471.

15. Liu K, Jiang L, Ruan J, et al. The role of dual energy CT in evaluating hemorrhagic complications at different stages after thrombectomy. Front Neurol. 2020; 11: 583411, doi: 10.3389/fneur.2020.583411, indexed in Pubmed: 33117268.

16. Almqvist H, Holmin S, Mazya MV. Dual energy CT after stroke thrombectomy alters assessment of hemorrhagic complications. Neurology. 2019; 93(11): e1068-e1075, doi: 10.1212/ WNL.0000000000008093, indexed in Pubmed: 31409735.

17. Tijssen MPM, Hofman PAM, Stadler AAR, et al. The role of dual energy CT in differentiating between brain haemorrhage and contrast medium after mechanical revascularisation in acute ischaemic stroke. Eur Radiol. 2014; 24(4): 834-840, doi: 10.1007/s00330-0133073-x, indexed in Pubmed: 24258277.

18. Chen S, Zhang J, Quan X, et al. Diagnostic accuracy of dual-energy computed tomography to differentiate intracerebral hemorrhage from contrast extravasation after endovascular thrombectomy for acute ischemic stroke: systematic review and meta-analysis. Eur Radiol. 2021 [Epub ahead of print], doi: 10.1007/s00330-021-08212-1, indexed in Pubmed: 34327578.

19. Bonatti M, Lombardo F, Zamboni GA, et al. lodine extravasation quantification on dual-energy CT of the brain performed after mechanical thrombectomy for acute ischemic stroke can predict hemorrhagic complications. AJNR Am J Neuroradiol. 2018; 39(3): 441-447, doi: 10.3174/ajnr.A5513, indexed in Pubmed: 29348131.

20. Almqvist H, Almqvist NS, Holmin S, et al. Dual-Energy CT follow-up after stroke thrombolysis alters assessment of hemorrhagic complications. Front Neurol. 2020; 11: 357, doi: 10.3389/fneur.2020.00357, indexed in Pubmed: 32508735. 